\title{
Microwave Zeeman Spectrum of Hypochlorous Acid
}

\author{
Masao Suzuki * and Antonio Guarnieri \\ Abteilung Chemische Physik im Institut für Physikalische Chemie der Universität Kiel
}

(Z. Naturforsch. 30 a, 497-505 [1975] ; received February 19, 1975)

\begin{abstract}
The microwave-Zeeman-spectrum of $\mathrm{HOCl}$ has been investigated with magnetic fields up to $25 \mathrm{kG}$. A method is described to perform the calculation of the Zeeman parameters independent from rotational constants and quadrupole coupling constants.
\end{abstract}

\section{Introduction}

In the recent years many investigations of the rotational Zeeman-effect have been reported. Among the 'molecules of different kinds are very few containing a chlorine atom ${ }^{1-4}$. We report the results of the Zeeman studies of the $\mathrm{HOCl}$ molecule.

Other asymmetric-top molecules containing chlorine are under investigation in this laboratory.

We started with this simple triatomic molecule, as the structure has been determined with a good approximation ${ }^{5}$. We hope further that quantum chemical calculations may be performed for this simple molecule.

\section{Experimental}

Hypochlorous acid ( $\mathrm{HOCl})$ was prepared condensing $\mathrm{Cl}_{2} \mathrm{O}(0.5 \mathrm{ml})$ on ice $(0.5 \mathrm{ml})$ at liquid nitrogen temperature.

The sample was then permitted to warm up to -20 to $-30{ }^{\circ} \mathrm{C}$ and a slow reaction took place at the contact surface until the equilibrium mixture $\mathrm{Cl}_{2} \mathrm{O}+\mathrm{H}_{2} \mathrm{O} \rightleftarrows 2 \mathrm{HOCl}$ was obtained. The sample was then stored in a bath at $-80{ }^{\circ} \mathrm{C}$ : a decomposition could not be detected at this temperature.

For the microwave measurements $\mathrm{HOCl}$ developing from the equilibrium mixture flowed continously through the waveguide at a pressure of approximately 4 mTorr and a temperature of $-23^{\circ} \mathrm{C}$.

The spectrometer used was a conventional $33 \mathrm{kHz}$ Stark-modulated one described elsewhere ${ }^{6}$.

The frequency sources were conventional BWO's up to $40 \mathrm{GHz}$. For the frequency range above $50 \mathrm{GHz}$ the second harmonic of a $30 \mathrm{~V} 12 \mathrm{OKI}$ Klystron was generated using a cross waveguide harmonic multiplier provided with a Schottky-barrier mixer diode PI 1909.

\footnotetext{
* On leave from Tokyo Institute of Technology, O-okayamaMeguro-ku - Tokyo/Japan.
}

\section{Spectrum}

HOCl is a prolate-top molecule with rotational constants $A=607 \mathrm{GHz}^{7}, B=15.11750 \mathrm{GHz}$ and $C=14.72504 \mathrm{GHz}^{5}$. Only an investigation of the $\mu_{a}$-type spectrum can be performed with our present apparatus. The measured lines are: $0_{00}-1_{01}, 1_{11}-$ $2_{12}$ and $1_{10}-2_{11}$. Due to the high values of the quadrupole coupling constants of chlorine $\left(\chi_{a a}\right.$ $\left.=-121.91, \chi_{b b}=59.61\right)$ and the relatively low magnetic field of $30 \mathrm{KG}$ only the coupled case could be investigated.

The appropriate Hamiltonian for the HOCl-molecule is in this case ${ }^{8}$ :

$$
\begin{aligned}
\mathcal{H} & =\mathcal{H}_{\text {rot }}+\mathcal{H}_{\text {mag }}+\mathcal{H}_{\mathrm{Q}}, \\
\mathcal{H}_{\text {mag }} & =\mathcal{H}_{\text {mag }}^{(\mathrm{g})}+\mathcal{H}_{\text {mag }}^{(\mathrm{s})}+\mathcal{H}_{\text {mag }}^{(\mathrm{I})} .
\end{aligned}
$$

Where $\mathcal{H}_{\text {rot }}$ is the usual zero-field rigid rotor Hamiltonian, $\mathcal{H}_{Q}$ is the nuclear quadrupole coupling Hamiltonian for the $\mathrm{Cl}$ atom and $\mathcal{H}_{\mathrm{mag}}$ describes the Zeeman effect which has three contributions: the rotational Zeeman-effect described by $\mathcal{H}_{\text {mag }}^{(\mathrm{g})}$ containing the molecular $g$-factors, the Zeeman effect originating from the induced magnetic moment described by $\mathcal{H}_{\text {mag }}^{(\mathrm{s})}$ containing the molecular mag. netic susceptibility tensor and the Zeeman effect of the chlorine nucleus described by $\mathcal{H}_{\text {mag }}^{(\mathrm{I})}$ containing the nuclear $g$-factor $g_{l}$.

Considering that the splitting due to the Zeeman effect at field strengths from 5 to $30 \mathrm{KG}$ is less than or at most equal to the quadrupole coupling splitting of the chlorine atom, the cases of low and intermediate field have to be used for the calculation of the energy levels.

The combined Zeeman-quadrupole effect can be described with the basis function $\left|J, \tau, I, F, M_{\mathrm{F}}\right\rangle$ or $\left|J, \tau, I, M_{J}, M_{l}\right\rangle$.

The first one is more adapted to the case of low fields, the second one for the case of intermediate and high fields. In the $\left|J, \tau, I, M_{l}, M_{J}\right\rangle$-basis only 
a $(J, \tau, I / J, \tau, I)$ submatrix will be considered. $\mathcal{H}_{\text {rot }}$ is diagonal in this basis. The contributions of $\mathcal{H}_{\mathrm{mag}}$ will be considered only to first order. The $(J, \tau, I /$ $J, \tau, I)$ submatrix has the following elements:

$$
\left\langle J, \tau, I, M_{J}, M_{I}\left|\mathcal{H}_{\mathrm{rot}}+\mathcal{H}_{\mathrm{mag}}\right| J, \tau, I, M_{J}, M_{l}\right\rangle=E_{\mathrm{rot}}+E_{\mathrm{mag}}^{(\mathrm{g})}+E_{\mathrm{mag}}^{(\mathrm{S})}+E_{\mathrm{mag}}^{(\mathrm{I})}
$$

where $E_{\text {rot }}$ are the usual asymmetric rotor eigenvalues

$$
\begin{gathered}
E_{\mathrm{mag}}^{\mathrm{g})}=-\mu_{0} \frac{M_{J} H_{\mathrm{z}}}{J(J+1)} \sum_{g} g_{g g}\left\langle J_{g}{ }^{2}\right\rangle \\
E_{\mathrm{mag}}^{(\mathrm{S})}=-1 / 2 \chi H_{\mathrm{z}}{ }^{2}-\left\{\frac{3 M_{J}{ }^{2}-J(J+1)}{3(2 J-1)(2 J+3) J(J+1)} \sum_{g}\left(3 \chi_{g g}-3 \chi\right)\left\langle J_{g}{ }^{2}\right\rangle\right\} \\
E_{\mathrm{mag}}^{(\mathrm{I})}=-\mu_{0} g_{I} M_{I} H_{\mathrm{z}} \\
\left\langle J, \tau, I, M_{J}, M_{I}\left|\mathcal{H}_{\mathrm{Q}}\right| J, \tau, I, M_{J}^{\prime}, M_{I}^{\prime}\right\rangle=\frac{C}{2}\left\{J(J+1)-3 M_{J}{ }^{2}\right\}\left\{I(I+1)-3 M_{I}{ }^{2}\right\} \cdot \delta_{M_{J^{\prime}} M_{J}} \delta_{M_{I^{\prime}} M_{I}} \\
+\frac{3}{4} C\left(2 M_{J} \pm 1\right)\left(2 M_{I} \mp 1\right) \sqrt{\left(J \mp M_{J}\right)\left(J \pm M_{J}+1\right)\left(I \pm M_{I}\right)\left(I \mp M_{I}+1\right)} \delta_{M_{J^{\prime}}, M_{J} \pm 1} \delta_{M_{I} M_{I}: \mp 1} \\
+\frac{3}{4} C \sqrt{\left(J \mp M_{J}\right)\left(J \mp M_{J}-1\right)\left(J \pm M_{J}+1\right)\left(J \pm M_{J}+2\right)\left(I \pm M_{I}\right)\left(I \pm M_{I}-1\right)\left(I \mp M_{I}+1\right)\left(I \mp M_{I}+2\right)} \\
\cdot \delta_{M_{J^{\prime}} M_{J} \pm 2} \delta_{M_{I^{\prime}} M_{I} \mp 2}
\end{gathered}
$$

the value of $C$ is:

$$
C=\frac{1}{J(J+1)(2 J-1)(2 J+3) I(2 I-1)} \sum_{g} \chi_{g g}^{(\mathrm{Cl})}\left\langle J_{g g}^{2}\right\rangle .
$$

$H_{z}$ is the applied magnetic field, $\mu_{0}$ the nuclear magneton, $g_{g g}$ the diagonal elements of the molecular $g$-tensor, $\chi_{g g}$ the diagonal elements of the magnetic susceptibility tensor, $\chi$ the bulk magnetic susceptibility, $g_{I}$ the $g$-factor of the chlorine nucleus, $\chi_{g g}^{(\mathrm{Cl})}$ the nuclear quadrupole coupling constants of the chlorine atom and $\left\langle J_{g}{ }^{2}\right\rangle$ the expectation value of the squared angular momentum operator for the state $J, \tau$.

In the basis $\left|J, \tau, I, F, M_{\mathrm{F}}\right\rangle$ the Hamiltonian $\mathcal{H}_{\mathrm{Q}}$ of the quadrupole coupling energy is diagonal in $M_{\mathrm{F}}=M_{J}+M_{I}$ and the matrix of the total Hamiltonian (1) has only diagonal elements.

In the Table 1 the submatrices for $J=0,1,2$ are given in detail with running $M_{J}$ and $M_{l}$.

$I$ is fixed to $3 / 2$ for chlorine, $E_{\text {rot }}$ and $-\frac{1}{2} \chi H_{z}{ }^{2}$ have been omitted and $M_{\mathrm{F}}$ is given in addition to $M_{J}$ and $M_{l}$ indicating that the matrix is diagonal in $M_{\mathrm{F}}$ in the $\left|J, \tau, I, F, M_{\mathrm{F}}\right\rangle$-basis. Due to the limitation in the strength of the applied magnetic field, it was not possible to reach the more simple case of high field condition.

For this reason the basis $\left|J, \tau, I, F, M_{\mathrm{F}}\right\rangle$ has been found more convenient for the characterisation of the energy levels $E\left(J, \tau, I, F, M_{\mathrm{F}}\right)$ and for comparison with the zero field values $E_{\text {rot }}(J, \tau)$

$$
\begin{gathered}
E\left(J, \tau, I, F, M_{\mathrm{F}}\right)=E_{\mathrm{rot}}(J, \tau)-\frac{1}{2} \chi H_{z}^{2} \\
-E^{\prime}\left(J, \tau, I, F, M_{\mathrm{F}}\right) .
\end{gathered}
$$

$E^{\prime}\left(J, \tau, I, F, M_{\mathrm{F}}\right)$ are the eigenvalues of matrices given in Table 1 .

To avoid the use of inaccurate rotational constants and of the quadrupole coupling constants of chlorine for the determination of all Zeeman parameters, a method has been developed which uses the frequency differences between measured Zeeman satellites.

Simple relations have been obtained relating the frequency differences and the $g$-factors only or the magnetic susceptibility anisotropies only.

\begin{tabular}{llcc}
\hline & & $M_{\mathrm{F}}= \pm 3 / 2$ & $M_{\mathrm{F}}= \pm 1 / 2$ \\
\cline { 3 - 4 } & & $M_{J}=0 ; M_{I}= \pm 3 / 2$ & $M_{J}=0 ; M_{I}= \pm 1 / 2$ \\
\hline$M_{\mathrm{F}}= \pm 3 / 2$ & $M_{J=0}$ & $\mp 3 / 2 \mu_{0} g_{I} H_{z}$ & 0 \\
\hline$M_{I}= \pm 3 / 2$ & 0 & $\mp 1 / 2 \mu_{0} g_{I} H_{z}$ \\
\hline
\end{tabular}

Table 1 a. Submatrix for $\boldsymbol{J}=\mathbf{0}$. 
Table $1 \mathrm{~b}$. Part of the submatrix for $J=1$ with the elements used in this work.

\begin{tabular}{|c|c|c|c|c|c|}
\hline & & $M_{\mathrm{F}}= \pm 5 / 2$ & 1 & \multicolumn{2}{|c|}{$M_{\mathrm{F}}= \pm 3 / 2$} \\
\hline & & $M_{J}= \pm 1 ; \quad M_{I}= \pm 3 / 2$ & 1 & $M_{J}= \pm 1 ; M_{I}= \pm 1 / 2$ & $M_{J}=0 ; M_{I}= \pm 3 / 2$ \\
\hline$M_{\mathrm{F}}= \pm 5 / 2$ & $\begin{array}{l}M_{J}= \pm 1 \\
M_{I}= \pm 3 / 2\end{array}$ & $\begin{array}{l}\mp 3 / 2 \mu_{0} g_{I} H_{z} \\
\mp 1 / 2 \mu_{0} \sum_{g g} g_{g g}\left\langle J_{g}{ }^{2}\right\rangle_{1 \tau} H_{z} \\
-H_{z^{2}} / 30 \sum_{g}(3 \chi g g-3 \chi)\left\langle J_{g^{2}}\right\rangle_{1 \tau} \\
+1 / 20 \sum_{g} \chi_{g g}^{(\mathrm{C} 1)}\left\langle J_{g^{2}}\right\rangle_{1 \tau}\end{array}$ & & 0 & 0 \\
\hline \multirow{2}{*}{$M_{\mathrm{F}}= \pm 3 / 2$} & $\begin{array}{l}M_{J}= \pm 1 \\
M_{l}= \pm 1 / 2\end{array}$ & 0 & & $\begin{array}{l}\mp 1 / 2 \mu_{0} g_{I} H_{z} \\
\mp 1 / 2 \mu_{0} \sum_{g} g_{g g}\left\langle J_{g^{2}}\right\rangle_{1 \tau} H_{z} \\
-H_{z^{2}} / 30 \sum_{g}(3 \chi g g-3 \chi)\left\langle J_{g}^{2}\right\rangle_{1 \tau} \\
-1 / 20 \sum_{g} \chi_{g g}^{\left(\mathrm{C}_{1}\right)}\left\langle J_{g^{2}}\right\rangle_{1 \tau}\end{array}$ & $\frac{\sqrt{6}}{20} \sum_{g} \chi_{g g}^{\left(\mathrm{C}_{11}\right)}\left\langle J_{g}^{2}\right\rangle_{1 \tau}$ \\
\hline & $\begin{array}{l}M_{J}=0 \\
M_{I}= \pm 3 / 2\end{array}$ & 0 & - & $\frac{\sqrt{6}}{20} \sum_{g} \chi_{g g}^{\left(\mathrm{C}_{1}\right)}\left\langle J_{g}{ }^{2}\right\rangle_{1}$ & $\begin{array}{l} \pm 3 / 2 \mu_{0} g_{I} H_{z} \\
\left.+H_{z}^{2} / 15 \sum_{g}^{(3} \chi_{g g}-3 \chi\right)\left\langle J_{g^{2}}\right\rangle_{1 \tau} \\
-1 / 10 \sum_{g} \chi_{g g}^{(\mathrm{C} 1)}\left\langle J_{g^{2}}\right\rangle_{1 \tau}\end{array}$ \\
\hline
\end{tabular}

Table 1 c. Part of the submatrix for $J=2$ with the elements used in this work.

\begin{tabular}{|c|c|c|c|c|}
\hline & & $M_{\mathrm{F}}= \pm 7 / 2$ & \multicolumn{2}{|c|}{$M_{\mathrm{F}}= \pm 5 / 2$} \\
\hline & & $M_{J}= \pm 2 ; \quad M_{I}= \pm 1 / 3$ & $M_{J}= \pm 2 ; M_{I}= \pm 1 / 2$ & $M_{J}= \pm 1 ; M_{I}= \pm 3 / 2$ \\
\hline$M_{\mathrm{F}}= \pm 7 / 2$ & $\begin{array}{l}M_{J}= \pm 2 \\
M_{I}= \pm 3 / 2\end{array}$ & $\begin{array}{l}\mp 3 / 2 \mu_{0} g_{I} H_{z} \\
\mp 1 / 3 \mu_{0} \underset{g}{\sum_{g g g}\left\langle J_{g^{2}}\right\rangle_{2 \tau} H_{z}} \\
-H_{\mathrm{z}}^{2} / 63 \sum_{g}\left(3 \chi_{g g}-3 \chi\right)\left\langle J_{g^{2}}\right\rangle_{2 \tau} \\
+1 / 42 \sum_{g} \chi_{g g}^{-\mathrm{Cl})}\left\langle J_{g^{2}}\right\rangle_{2 \tau}\end{array}$ & 0 & 0 \\
\hline \multirow{2}{*}{$M_{\mathrm{F}}= \pm 5 / 2$} & $\begin{array}{c}M_{J}= \pm 2 ; \\
M_{I}= \pm 1 / 2\end{array}$ & 0 & $\begin{array}{l}\mp 1 / 2 \mu_{0} g_{I} H_{z} \\
\mp 1 / 3 \mu_{0} H_{z} \sum_{g} g_{g g}\left\langle J_{g^{2}}\right\rangle_{2 \tau} \\
-H_{z}^{2} / 63 \sum_{g}(3 \chi g g-3 \chi)\left\langle J_{g^{2}}\right\rangle_{2 \tau} \\
-1 / 42 \sum_{g} \chi_{9 g}^{(\mathrm{Cl} 1)}\left\langle J_{g^{2}}\right\rangle_{2 \tau}\end{array}$ & $\frac{\sqrt{3}}{42} \sum_{g} \chi_{g g}^{(\mathrm{C} 1)}\left\langle J_{g}^{2}\right\rangle_{2 \tau}$ \\
\hline & $\begin{array}{c}M_{J}= \pm 1 \\
M_{I}= \pm 3 / 2\end{array}$ & 0 & $\frac{\sqrt{3}}{42} \sum_{g} \chi_{g g}^{\left(\mathrm{C}_{1}\right)}\left\langle J_{g^{2}}\right\rangle_{2 \tau}$ & $\begin{array}{l}\mp 3 / 2 \mu_{0} g_{I} H_{z} \\
\mp 1 / 6 \mu_{0} H_{z} \sum_{g} g_{g g}\left\langle J_{g^{2}}\right\rangle_{2 \tau} \\
+H_{z}^{2} / 126 \sum_{g}^{\prime} \cdot\left(3 \chi_{g g}-3 \chi\right)\left\langle J_{g^{2}}\right\rangle_{2 \tau} \\
-1 / 84 \sum_{g} \chi_{g \delta}^{(\mathrm{Cl})}\left\langle J_{g^{2}}\right\rangle_{2 \tau}\end{array}$ \\
\hline
\end{tabular}


Thus the values of these parameters are determined from experimental results directly without using involved fitting procedures.

The following procedure is used in the case of parallel fields with $\Delta M_{\mathrm{F}}=0$ : The transition fre- quency from the level $E\left(J, \tau, I, F, M_{\mathrm{F}}\right)$ to the level $E^{\prime}\left(J^{\prime}, \tau^{\prime}, I^{\prime}, F, M_{\mathrm{F}}{ }^{\prime}\right)$ is expressed by the notation $v\left(J_{\tau} \rightarrow J_{\tau^{\prime}}^{\prime} ; F \rightarrow F^{\prime} ; M_{\mathrm{F}} ; H_{z}\right)$. Further we use the sum of the transition frequencies

and

$$
\begin{aligned}
& v\left[J_{\tau} \rightarrow(J+1)_{\tau^{\prime}} ; F=J+I \rightarrow F^{\prime}=F+1 ; M_{\mathrm{F}}=F ; H_{z}\right] \\
& v\left[J_{\tau} \rightarrow(J+1)_{\tau^{\prime}} ; F=J+I \rightarrow F^{\prime}=F ; M_{\mathrm{F}}=F ; H_{z}\right]
\end{aligned}
$$

in the following formula

$\nu^{ \pm}\left(M_{\mathrm{F}}= \pm F\right)$

$=\frac{v\left(J_{\tau} \rightarrow J_{\tau^{\prime}}^{\prime} ; F=J+I \rightarrow F^{\prime}=F+1 ; M_{\mathrm{F}}= \pm F ; H_{z}\right)+v\left(J_{\tau} \rightarrow J_{\tau^{\prime}}^{\prime} ; F=J+I \rightarrow F^{\prime}=F ; M_{\mathrm{F}}= \pm F ; H_{z}\right)}{2}$.

Now it is easy to see that

$$
v^{-}\left(M_{\mathrm{F}}=-F\right)-v^{+}\left(M_{\mathrm{F}}=+F\right)=D_{v}^{-}=\mu_{0} \frac{H_{z}}{h} \Delta \quad \text { where } \quad \Delta=f\left(g_{l}, g_{a a}, g_{b b}, g_{c c}\right)
$$

is only a function of $g_{l}$ and $g$-factors.

For the susceptibility anisotropies the following formula was developed:

$$
\left[v^{-}\left(M_{\mathrm{F}}=-F\right)+v^{+}\left(M_{\mathrm{F}}=+F\right)\right]_{H_{z} \neq 0}-\left[v^{-}\left(M_{\mathrm{F}}=-F\right)+v^{+}\left(M_{\mathrm{F}}=+F\right)\right]_{H_{z}=0}=D_{v}^{+}=\frac{H_{z}^{2}}{h} S
$$

where

$$
S=f(\alpha, \beta), \quad \alpha=2 \chi_{a a}-\chi_{b b}-\chi_{c c} \quad \text { and } \beta=2 \chi_{b b}-\chi_{c c}-\chi_{a a} .
$$

Both $\Delta$ and $S$ functions are given in Table 2.

In the case of perpendicular field conditions $\left(\Delta M_{\mathrm{F}}= \pm 1\right)$ the following formulas have been found

$$
\begin{aligned}
& v\left[J_{\tau} \rightarrow(J+1)_{\tau^{\prime}} ; F=\right.\left.J \rightarrow F^{\prime}=F+1 ; M_{\mathrm{F}}=-F \rightarrow M_{\mathrm{F}}{ }^{\prime}=-(F+1) ; H_{z}\right] \\
& \quad-v\left[J_{\tau} \rightarrow(J+1)_{\tau^{\prime}} ; F=J+I \rightarrow F^{\prime}=F+1 ; M_{\mathrm{F}}=F \rightarrow M_{\mathrm{F}}{ }^{\prime}=F+1 ; H_{z}\right] \\
&= {\left[v\left(\Delta M_{\mathrm{F}}=-1\right)-v\left(\Delta M_{\mathrm{F}}=+1\right)\right]=D_{v}{ }^{-}=\mu_{0} \frac{H_{z}}{h} \Delta } \\
& {\left[v\left(\Delta M_{\mathrm{F}}=-1\right)+v\left(\Delta M_{\mathrm{F}}=+1\right)\right]_{H_{z} \neq 0}-\left[v\left(\Delta M_{\mathrm{F}}=-1\right)+v\left(\Delta M_{\mathrm{F}}=+1\right)\right]_{H_{z}=0}=D_{v}{ }^{+}=\frac{H_{z}{ }^{2}}{h} S . }
\end{aligned}
$$

Further, for the $0_{00}-1_{01}$ transition the formulas given in the appendix were developed.

Using the relations $(11,12),(13,14)$ and $(\mathrm{A} 1$, A 2) the values of the $g$-factors and of the magnetic susceptibility anisotropies could be obtained. The Zeeman splittings at several magnetic fields of those transitions used to derive the functions (see
Table 2) are given in Table 3 together with the $D_{v}^{+}$ and $D_{v}{ }^{-}$values.

The assignment was made observing the relative intensities and the frequency shifts of each Zeeman component. $D_{v}^{ \pm}$values smaller than $30 \mathrm{kHz}$ have been omitted, as the experimental errors of $D_{v}{ }^{ \pm}$ have been estimated to be about $\pm 20 \mathrm{kHz}$. From

Table 2. List of the obtained functions $\Delta_{i}\left(g_{I}, g_{a a}, g_{b b}, g_{c c}\right)$ and $S_{i}(\alpha, \beta)$. Nr. 1 and 2 refer to $\Delta M_{\mathrm{F}}=0$ case $\mathrm{Nr} .3,4$, 5 and 6 to the $\Delta M_{F}= \pm 1$ case.

\begin{tabular}{llllll}
\hline $\mathrm{i}$ & Transition & $F$ & $\left|M_{\mathrm{F}}\right| \rightarrow\left|M_{\mathrm{F}}\right|$ & $\Delta\left(g_{I}, g_{a a} g_{b b} g_{c c}\right)$ & $S(\alpha, \beta)$ \\
\hline 1 & $0_{00} \rightarrow 1_{01}$ & $3 / 2$ & $3 / 2 \rightarrow 3 / 2$ & $-g_{I}+1 / 2\left(g_{b b}+g_{c c}\right)$ & $-\alpha / 30$ \\
2 & $1_{11} \rightarrow 2_{12}$ & $5 / 2$ & $5 / 2 \rightarrow 5 / 2$ & $-g_{I}-1 / 2 g_{a a}+1 / 2 g_{b b}+g_{c c}$ & $(5 \alpha-9 \beta) / 210$ \\
3 & $0_{00} \rightarrow 1_{01}$ & $3 / 2$ & $3 / 2 \rightarrow 5 / 2$ & $g_{b b}+g_{c c}$ & $\alpha / 15$ \\
4 & $1_{11} \rightarrow 2_{12}$ & $5 / 2$ & $5 / 2 \rightarrow 7 / 2$ & $1 / 3\left(-g_{a a}+2 g_{b b}+5 g_{c c}\right)$ & $(10 \alpha+3 \beta) / 105$ \\
5 & $1_{10} \rightarrow 2_{11}$ & $5 / 2$ & $5 / 2 \rightarrow 7 / 2$ & $1 / 3\left(-g_{a a}+5 g_{b b}+2 g_{c c}\right)$ & $(7-3 \beta) / 105$ \\
6 & $0_{00} \rightarrow 1_{01}$ & $3 / 2$ & $1 / 2 \rightarrow 3 / 2$ & $g_{I}+1 / 2\left(g_{b b}+g_{c c}\right)$ & $-\alpha / 30$ \\
\hline
\end{tabular}


Table 3. Observed $D_{v}^{-}$and $D_{v}^{+}$values.

\begin{tabular}{|c|c|c|c|c|c|c|c|c|c|c|c|}
\hline$J \rightarrow J^{\prime}$ & $\begin{array}{l}\text { Field } \\
\text { (Gauss) }\end{array}$ & $F \rightarrow F^{\prime}$ & $M_{\mathrm{F}} \rightarrow M_{\mathrm{F}}^{\prime}$ & $\begin{array}{l}\text { Obs. Freq. } \\
\text { (MHz) }\end{array}$ & $F \rightarrow F^{\prime}$ & $M_{\mathrm{F}} \rightarrow M_{\mathrm{F}^{\prime}}$ & $\begin{array}{l}\text { Obs. Freq. } \\
\text { (MHz) }\end{array}$ & obs. $^{D_{v}^{-}(1}$ & $\begin{array}{l}\mathrm{Hz}) \\
\text { calc. * }\end{array}$ & $\begin{array}{l}D_{\nu}^{+} \\
\text {obs. }\end{array}$ & $\begin{array}{l}\text { Hz) } \\
\text { calc. * }\end{array}$ \\
\hline \multirow[t]{6}{*}{$0_{00} \rightarrow 1_{01}$} & 0.0 & $3 / 2 \rightarrow 5 / 2$ & & 29848.586 & $3 / 2 \rightarrow 3 / 2$ & & 29818.063 & & & & \\
\hline & 13023.1 & $3 / 2 \rightarrow 5 / 2$ & 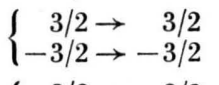 & $\begin{array}{l}29852.531 \\
29845.232\end{array}$ & $3 / 2 \rightarrow 3 / 2$ & $\left\{\begin{array}{rr}3 / 2 \rightarrow & 3 / 2 \\
-3 / 2 \rightarrow & -3 / 2\end{array}\right.$ & $\begin{array}{l}29820.279 \\
29815.328\end{array}$ & -6.125 & -6.129 & - & - \\
\hline & 19117.1 & $3 / 2 \rightarrow 5 / 2$ & $\left\{\begin{array}{r}3 / 2 \rightarrow 3 / 2 \\
-3 / 2 \rightarrow-3 / 2\end{array}\right.$ & $\begin{array}{l}29854.550 \\
29843.893\end{array}$ & $3 / 2 \rightarrow 3 / 2$ & $\left\{\begin{array}{rr}3 / 2 & \rightarrow \\
-3 / 2 & \rightarrow-3 / 2\end{array}\right.$ & $\begin{array}{l}29821.168 \\
29813.830\end{array}$ & -8.998 & -8.998 & 72 & 78 \\
\hline & 24078.9 & $3 / 2 \rightarrow 5 / 2$ & $\left\{\begin{array}{l}3 / 2 \rightarrow 3 / 2 \\
3 / 2 \rightarrow 3 / 2\end{array}\right.$ & $\begin{array}{l}29856.263 \\
29842.937\end{array}$ & $3 / 2 \rightarrow 3 / 2$ & $\left\{\begin{array}{rr}3 / 2 \rightarrow & 3 / 2 \\
-3 / 2 \rightarrow & -3 / 2\end{array}\right.$ & $\begin{array}{l}29821.851 \\
29812.504\end{array}$ & -11.337 & -11.334 & 129 & 124 \\
\hline & 9820.9 & $3 / 2 \rightarrow 5 / 2$ & $\left\{\begin{array}{r}3 / 2 \rightarrow 5 / 2 \\
-3 / 2 \rightarrow-5 / 2\end{array}\right.$ & $\begin{array}{l}29849.075 \\
29848.043\end{array}$ & & & & -1.032 & -1.031 & - & - \\
\hline & 14707.3 & $3 / 2 \rightarrow 5 / 2$ & 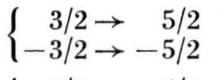 & $\begin{array}{l}29849.305 \\
29847.765\end{array}$ & & & & -1.540 & -1.543 & -102 & -104 \\
\hline & 21966.5 & $3 / 2 \rightarrow 5 / 2$ & $\left\{\begin{array}{r}3 / 2 \rightarrow 5 / 2 \\
-3 / 2 \rightarrow-5 / 2\end{array}\right.$ & $\begin{array}{l}29849.623 \\
29847.317\end{array}$ & & & & -2.305 & -2.305 & -232 & -232 \\
\hline & 14707.3 & $3 / 2 \rightarrow 5 / 2$ & $\left\{\begin{array}{rr}1 / 2 \rightarrow & 3 / 2 \\
-1 / 2 \rightarrow & -3 / 2\end{array}\right.$ & $\begin{array}{l}29846.942 \\
29850.995\end{array}$ & $3 / 2 \rightarrow 3 / 2$ & $\left\{\begin{array}{r}1 / 2 \rightarrow 3 / 2 \\
-1 / 2 \rightarrow-3 / 2\end{array}\right.$ & $\begin{array}{l}29814.395 \\
29821.070\end{array}$ & 5.364 & 5.364 & - & - \\
\hline \multirow[t]{6}{*}{$1_{11} \rightarrow 2_{12}$} & 0.0 & $5 / 2 \rightarrow 7 / 2$ & & 59295.709 & $5 / 2 \rightarrow 5 / 2$ & & 59280.108 & & & & \\
\hline & 6511.7 & $5 / 2 \rightarrow 7 / 2$ & 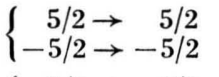 & $\begin{array}{l}59298.053 \\
59293.605\end{array}$ & $5 / 2 \rightarrow 5 / 2$ & $\left\{\begin{array}{rr}5 / 2 & \rightarrow 5 / 2 \\
-5 / 2 & \rightarrow-5 / 2\end{array}\right.$ & $\begin{array}{l}59282.592 \\
59277.425\end{array}$ & -4.808 & -4.812 & - & - \\
\hline & 13002.0 & $5 / 2 \rightarrow 7 / 2$ & $\left\{\begin{array}{rr}5 / 2 \rightarrow & 5 / 2 \\
-5 / 2 \rightarrow & -5 / 2\end{array}\right.$ & $\begin{array}{l}59300.525 \\
59291.630\end{array}$ & $5 / 2 \rightarrow 5 / 2$ & $\left\{\begin{array}{rr}5 / 2 & \rightarrow 5 / 2 \\
-5 / 2 & \rightarrow-5 / 2\end{array}\right.$ & $\begin{array}{l}59284.868 \\
59274.540\end{array}$ & -9.612 & -9.609 & - & - \\
\hline & 9824.3 & $5 / 2 \rightarrow 7 / 2$ & $\left\{\begin{array}{r}5 / 2 \rightarrow 7 / 2 \\
-5 / 2 \rightarrow-7 / 2\end{array}\right.$ & $\begin{array}{l}59297.075 \\
59294.324\end{array}$ & & & & -2.751 & -2.739 & - & - \\
\hline & 14702.9 & $5 / 2 \rightarrow 7 / 2$ & $\left\{\begin{array}{r}5 / 2 \rightarrow 7 / 2 \\
-5 / 2 \rightarrow-7 / 2\end{array}\right.$ & $\begin{array}{l}59297.710 \\
59293.629\end{array}$ & & & & -4.081 & -4.098 & -79 & -95 \\
\hline & 21966.1 & $5 / 2 \rightarrow 7 / 2$ & $\left\{\begin{array}{rr}5 / 2 \rightarrow & 7 / 2 \\
-5 / 2 \rightarrow & -7 / 2\end{array}\right.$ & $\begin{array}{l}59298.658 \\
59292.531\end{array}$ & & & & -6.127 & -6.121 & -229 & -222 \\
\hline \multirow[t]{4}{*}{$1_{10} \rightarrow 2_{11}$} & 0.0 & $5 / 2 \rightarrow 7 / 2$ & & 60077.657 & & & & & & & \\
\hline & 9833.2 & $5 / 2 \rightarrow 7 / 2$ & $\left\{\begin{array}{rr}5 / 2 & \rightarrow \\
-5 / 2 & \rightarrow-7 / 2 \\
-7 / 2\end{array}\right.$ & $\begin{array}{l}60079.065 \\
60076.211\end{array}$ & & & & -2.854 & -2.850 & - & - \\
\hline & 14704.2 & $5 / 2 \rightarrow 7 / 2$ & $\left\{\begin{array}{r}5 / 2 \rightarrow 7 / 2 \\
-5 / 2 \rightarrow-7 / 2\end{array}\right.$ & $\begin{array}{l}60079.737 \\
60075.465\end{array}$ & & & & -4.272 & -4.258 & -112 & -113 \\
\hline & 21968.7 & & $\left\{\begin{aligned} 5 / 2 & \rightarrow \\
-5 / 2 & \rightarrow-7 / 2\end{aligned}\right.$ & $\begin{array}{l}60080.703 \\
60074.357\end{array}$ & & & & -6.346 & -6.358 & -254 & -253 \\
\hline
\end{tabular}

* These values have been calculated by putting the values listed in Table 3 into the relation,

$$
D_{\nu}{ }^{-}=0.76227_{2} \times 10^{-3} \times \Delta \times H_{z} \text { and } D_{v}{ }^{+}=\frac{1}{3.99027} \times S \times H_{z^{2}} .
$$


$D_{v}{ }^{+}$and $D_{v}^{-}$-values the functions $\Delta$ and $S$ could be estimated using a least square fitting procedure. They are listed in Table 4.

Table 4. The value of the functions shown in Table 2.

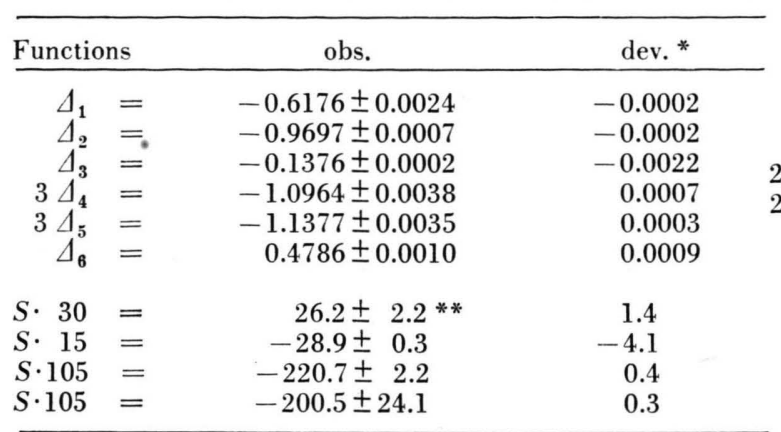

* dev. are the differences between the observed and the calculated values.

* The units are $10^{-6} \mathrm{erg} / \mathrm{G}^{2} \cdot \mathrm{mole}$.

The signs of the $g$-factors, as listed in Table 6 could be determined using the knowledge that the sign of the $g_{l}$-value of chlorine is positive ${ }^{\mathbf{1 0}}$.

In this case no reference to the magnitude of the molecular electric quadrupole moment or the sign of the second moment of the electric charge is necessary.

The electric quadrupole coupling constants of the chlorine atom were remeasured from the frequency splitting of the $0_{00}-1_{01}, 1_{11}-2_{12}$ and $1_{10}-2_{11}$ transitions at zero magnetic field as reported in Table 5. The results, listed in Table 6, agree with the values reported by Mirri et al. ${ }^{5}$ within experimental errors. For a final check the frequency split-

Table 6. Chlorine nuclear $g_{l}$-factor, molecular $g$-values, magnetic susceptibility anisotropy parameters and nuclear quadrupole constants of chlorine.

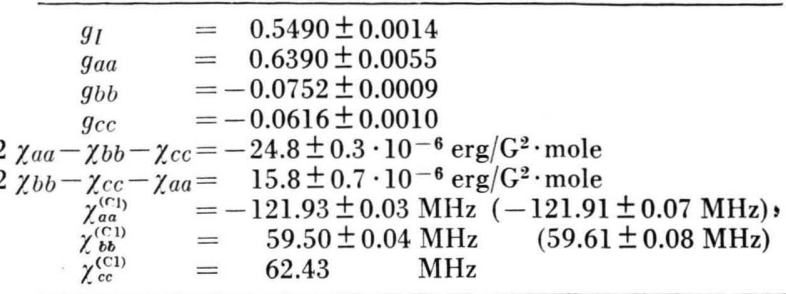

The values in parenthesis are given in a preceeding work from Mirri et al. Our values fit the measurements of this paper in a better way.

tings observed for the $0_{00}-1_{01}, 1_{11}-2_{12}, 1_{10}-2_{11}$ and $1_{01}-2_{02}$ transitions are listed again in Table 7 for both $\Delta M_{\mathrm{F}}=0$ and $\Delta M_{\mathrm{F}}= \pm 1$ cases and com. pared with calculated values which were obtained by solving the Hamiltonian (2). In these calculations the values reported by Ashby ${ }^{8}$ were taken for the rotational constant $A$ and those reported by Mirri et al. ${ }^{5}$ for the constants $B$ and $C$.

\section{Molecular Electric Quadrupole Moment}

Combining the molecular $g$-values, the magnetic susceptibility anisotropies and the rotational constants, the diagonal elements of the molecular electric quadrupole moment tensor can be calculated using Eq. (15)

\begin{tabular}{|c|c|c|c|c|}
\hline \multicolumn{5}{|c|}{$Q_{a a}=-\frac{\hbar|e|}{8 \pi M_{\mathrm{p}}}\left(\frac{2 g_{a a}}{A}-\frac{g_{b b}}{B}-\frac{g_{c c}}{C}\right)-$} \\
\hline$J_{K-K^{+}} \rightarrow J_{K-K^{+}}^{\prime}$ & corr. * & $F \rightarrow F^{\prime}$ & obs. & calc. \\
\hline $0_{00} \rightarrow 1_{01}$ & $\begin{array}{c}29842.461 \\
\pm 0.011\end{array}$ & $\left\{\begin{array}{l}3 / 2 \rightarrow 1 / 2 \\
3 / 2 \rightarrow 5 / 2 \\
3 / 2 \rightarrow 3 / 2\end{array}\right.$ & $\begin{array}{l}29872.925 \\
29848.586 \\
29818.063\end{array}$ & $\begin{array}{l}29872.943 \\
29848.557 \\
29818.075\end{array}$ \\
\hline $1_{11} \rightarrow 2_{12}$ & $\begin{array}{r}59288 \\
\pm 0 .\end{array}$ & $\left\{\begin{array}{l}1 / 2 \rightarrow 1 / 2 \\
1 / 2 \rightarrow 3 / 2 \\
5 / 2 \rightarrow 7 / 2 \\
5 / 2 \rightarrow 5 / 2 \\
3 / 2 \rightarrow 3 / 2 \\
3 / 2 \rightarrow 5 / 2\end{array}\right.$ & $\begin{array}{l}59318.759 \\
59303.141 \\
59295.709 \\
59280.108 \\
59276.366 \\
59265.229\end{array}$ & $\begin{array}{l}59318.756 \\
59303.149 \\
59295.708 \\
59280.101 \\
59276.373 \\
59265.226\end{array}$ \\
\hline $1_{10}-$ & $\begin{array}{r}60070 \\
\pm 0.0\end{array}$ & $\left\{\begin{array}{l}1 / 2 \rightarrow 1 / 2 \\
1 / 2 \rightarrow 3 / 2 \\
5 / 2 \rightarrow 7 / 2 \\
5 / 2 \rightarrow 5 / 2 \\
3 / 2 \rightarrow 3 / 2 \\
3 / 2 \rightarrow 5 / 2\end{array}\right.$ & $\begin{array}{l}60100.719 \\
60085.880 \\
60077.657 \\
60062.733 \\
60057.764 \\
60047.144\end{array}$ & $\begin{array}{l}60100.738 \\
60085.863 \\
60077.628 \\
60062.752 \\
60057.771 \\
60047.145\end{array}$ \\
\hline $1_{01} \rightarrow 2_{02}$ & $\begin{array}{c}59684.064 \\
\pm 0.013\end{array}$ & $\left\{\begin{array}{l}3 / 2 \rightarrow 3 / 2 \\
5 / 2 \rightarrow 5 / 2\end{array}\right.$ & $\begin{array}{l}59708.463 \\
59656.181\end{array}$ & $\begin{array}{l}59708.450 \\
59656.195\end{array}$ \\
\hline
\end{tabular}

Table 5. Transition frequencies $(\mathrm{MHz})$ at zero magnetic fields.

* corr. are the corrected frequencies for the nuclear quadrupole splittings. 


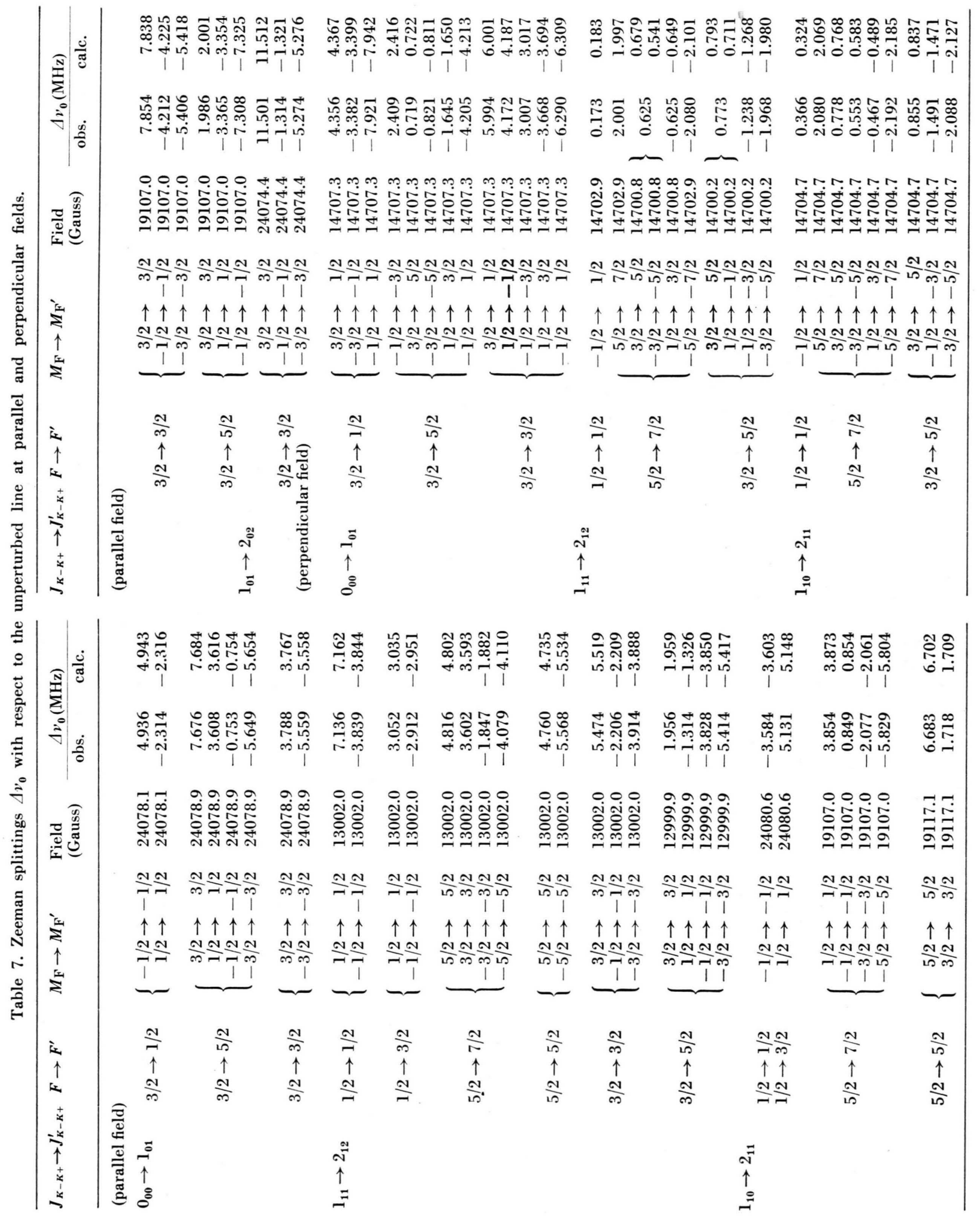


and cyclic, where $|e|$ is the electronic charge, $M_{\mathrm{p}}$ the proton mass, $m$ the electron mass. $A, B$ and $C$ are rotational constants, $g_{g g}$ the molecular $g$-factors and $\chi_{g g}$ the magnetic susceptibilities. The $Q_{g g}$ are given in Table 8.

The second moment of the electron charge can be calculated in the form given as follows:

$$
\begin{gathered}
\left\langle b^{2}\right\rangle-\left\langle a^{2}\right\rangle=\sum_{n} Z_{n}\left(b_{n}{ }^{2}-a_{n}{ }^{2}\right)+\frac{\hbar}{4 \pi M_{\mathrm{p}}}\left(\frac{g_{b b}}{B}-\frac{g_{a a}}{A}\right)+\frac{4 m c^{2}}{3 e^{2}}\left[\left(2 \chi_{b b}-\chi_{a a}-\chi_{c c}\right)-\left(2 \chi_{a a}-\chi_{b b}-\chi_{c c}\right)\right] \\
\left\langle a^{2}\right\rangle=\left\langle 0\left|\Sigma a_{i}{ }^{2}\right| 0\right\rangle
\end{gathered}
$$

Table 8. Diagonal elements of the molecular electric quadrupole moment tensor.

$$
\begin{aligned}
& Q_{a a}=+(0.50 \pm 0.35) \cdot 10^{-26} \mathrm{esu} \\
& Q_{b b}=+(0.74 \pm 0.67) \cdot 10^{-26} \mathrm{esu} \\
& Q_{c c}=-(1.24 \pm 1.02) \cdot 10^{-26} \mathrm{esu}
\end{aligned}
$$

where $Z_{n}$ is the atomic number of the nth nucleus, $a_{n}$ and $b_{n}$ are its coordinates in the principal axes, $a_{i}$ is the coordinate of the $i^{\text {th }}$ electron.

$\left\langle c^{2}\right\rangle-\left\langle b^{2}\right\rangle$ and $\left\langle a^{2}\right\rangle-\left\langle c^{2}\right\rangle$ are obtained by cyclic permutations.

Using nuclear second moments calculated from the known structure of $\mathrm{HOCl}^{5}$ :

$$
\begin{aligned}
& \sum_{n} Z_{n} a_{n}{ }^{2}=(17.41 \pm 0.04) \cdot 10^{-16} \mathrm{~cm}^{2}, \\
& \sum_{n} Z_{n} b_{n}{ }^{2}=(0.81 \pm 0.03) \cdot 10^{-16} \mathrm{~cm}^{2}, \\
& \sum_{n} Z_{n} c_{n}{ }^{2}=0.0
\end{aligned}
$$

and the empirical rule given in (9) for the estimation of $\left\langle c^{2}\right\rangle$ from free atom values, one gets the values given in Table 9. Further the diagonal elements of the paramagnetic susceptibility tensor and of the diamagnetic susceptibility tensor given by the following formulas, could be calculated.

$$
\chi_{a a}^{p}=-\left(\frac{e^{2} N}{2 m c^{2}}\right)\left[\left(\frac{\hbar g_{a a}}{8 \pi A M_{\mathrm{p}}}\right)-\frac{1}{2} \sum_{n} Z_{n}\left(b_{n}{ }^{2}+c_{n}{ }^{2}\right)\right]
$$

\begin{tabular}{|c|c|}
\hline$\left\langle a^{2}\right\rangle$ & $21.3 \pm 0.5$ \\
\hline$\left\langle b^{2}\right\rangle$ & $4.8 \pm 0.5$ \\
\hline$\left\langle c^{2}\right\rangle$ & $3.9( \pm 0.3) *$ \\
\hline$\left\langle\chi_{a a}^{d}\right\rangle$ & $-(36.7 \pm 3.2)$ \\
\hline$\left\langle\lambda_{b b}^{d}\right\rangle$ & $-(106.3 \pm 3.2)$ \\
\hline$\left\langle\eta_{c c}^{p}\right\rangle$ & $-(110.4 \pm 3.9)$ \\
\hline$\left\langle\chi_{a a}^{p}\right\rangle$ & $1.2 \pm 0.2$ \\
\hline$\left\langle\chi_{b b}^{a}\right\rangle$ & $84.4 \pm 0.3$ \\
\hline$\left\langle\chi_{c c}^{p}\right\rangle$ & $86.2 \pm 0.4$ \\
\hline$\chi_{a a}$ & $-(35.5 \pm 3.4)$ \\
\hline$\chi_{b b}$ & $-(21.9 \pm 3.5)$ \\
\hline$\chi_{c c}$ & $-(24.2 \pm 4.3)$ \\
\hline$\chi=1 / 3\left(\chi_{a a}+\chi_{b b}+\chi_{c c}\right)$ & $-(27.2 \pm 3.7)$ \\
\hline
\end{tabular}

$\chi_{a a}^{d}=-\left(\frac{e^{2} N}{4 m c^{2}}\right)\left\langle 0\left|\sum\left(b^{2}+c^{2}\right)\right| 0\right\rangle$.

Table 9 lists all the calculated parameters. As the bulk magnetic susceptibility is the sum of a paramagnetic and a diamagnetic term it can be now evaluated to be

$$
\chi=-(27.2 \pm 3.7) \cdot 10^{-6} \mathrm{erg} / \mathrm{G}^{2} \cdot \text { mole } .
$$

Table 9. Calculated Zeeman-parameters.

\section{Appendix}

For the transition $0_{00} \rightarrow 1_{01}(i=6$ in Table 2) the following formulas were developed:

$$
\begin{gathered}
v\left(F=3 / 2 \rightarrow F^{\prime}=5 / 2 ; M_{\mathrm{F}}=-1 / 2 \rightarrow M_{\mathrm{F}}^{\prime}=-3 / 2 ; H_{z}\right)=v_{1}, \\
v\left(F=3 / 2 \rightarrow F^{\prime}=3 / 2 ; M_{\mathrm{F}}=-1 / 2 \rightarrow M_{\mathrm{F}}^{\prime}=-3 / 2 ; H_{z}\right)=v_{2}, \\
v\left(F=3 / 2 \rightarrow F^{\prime}=5 / 2 ; M_{\mathrm{F}}=1 / 2 \rightarrow M_{\mathrm{F}}^{\prime}=3 / 2 ; H_{z}\right)=v_{3}, \\
v\left(F=3 / 2 \rightarrow F^{\prime}=3 / 2 ; M_{\mathrm{F}}=1 / 2 \rightarrow M_{\mathrm{F}}^{\prime}=3 / 2 ; H_{z}\right)=v_{4} ; \\
D_{v}{ }^{-}=\frac{v_{1}+v_{2}}{2}-\frac{v_{3}+v_{4}}{2}=\mu_{0}\left(H_{z} / h\right) \Delta,
\end{gathered}
$$




$$
D_{v}^{+}=\left[\frac{v_{1}+v_{2}}{2}+\frac{v_{3}+v_{4}}{2}\right]_{H_{2} \neq 0}-\left[\frac{v_{1}+v_{2}}{2}+\frac{v_{3}+v_{4}}{2}\right]_{H_{z}=0}=\frac{H_{z}^{2}}{h} S .
$$

$\triangle$ and $S$ are listed in Table 2.

\section{Acknowledgements}

We wish to thank the Deutsche Forschungsgemeinschaft and the Fonds der Chemie for a post-

1 D. L. Vanderhart and W. H. Flygare, Mol. Physics 18, 77 [1970].

2 J. McGurk, C. L. Norris, H. L. Tigelaar, and W. H. Flygare, J. Chem. Phys. 58, 3118 [1973].

3 J. J. Ewing, H. L. Tigelaar, and W. H. Flygare, J. Chem. Phys. 56, 1957 [1972].

4 C. L. Allen and W. H. Flygare, Chem. Phys. Lett. 15, 461 [1972]. doctoral fellowship (M.S.) and for research funds. Calculations have been made with the PDP 10 of the Rechenzentrum der University of Kiel.

5 A. M. Mirri, F. Scappini, and G. Cazzoli, J. Mol. Spectry 38, 218 [1971].

6 D. Sutter, Z. Naturforsch. 26 a, 1644 [1971].

7 R. A. Ashby, J. Mol. Spectry 23, 439 [1967].

8 W. Hüttner and W. H. Flygare, J. Chem. Phys. 47, 4137 [1967].

9 T. D. Gierke, H. L. Tigelaar, and W. H. Flygare, J. Amer. Chem. Soc. 94, 330 [1972].

10 Y. Saito, Canadian J. Chem. 43, 2530 [1965]. 